\title{
El valor de la historia. Estudio de alternativas curriculares en Secundaria (3): aprender a pensar desde múltiples perspectivas
}

The value of history. Study of curricular alternatives at secondary school (3): learning to think from multiple perspectives

Javier Paricio Royo*

\section{Resumen}

Explorar y debatir múltiples perspectivas sobre los acontecimientos del pasado es una orientación curricular tan aceptada entre los investigadores, como poco frecuente en nuestras aulas de historia. Como en entregas anteriores de esta serie de artículos, se analiza aquí esta orientación curricular a la luz de las investigaciones y experiencias publicadas, tratando de esclarecer en qué consiste, sus claves, sus prácticas más habituales y por qué resulta valiosa para los estudiantes. La multiperspectividad está en la misma esencia de la historia como disciplina. Se opone a la memoria, caracterizada por relatos cerrados que imponen una única versión del pasado. En esta tensión entre disciplina y memoria, la práctica de la enseñanza de la historia ha apostado habitualmente por la segunda. Pero aprender a analizar, valorar y debatir las distintas versiones del mundo del pasado (y del presente) tiene mucho más valor curricular. Multiperspetividad no sólo significa comprender la verdadera naturaleza polémica, abierta y crítica de la historia, sino que es la misma base de la ciudadanía democrática.

Palabras clave: Multiperspectiva. Perspectiva histórica. Historia controvertida. Pensamiento histórico. Diseño curricular. Enseñanza Secundaria.

\begin{abstract}
As a curricular orientation, discussing multiple perspectives on past events is as accepted among researchers as it is rare in our history classrooms. Following the trail of previous issues in this series of articles, this curricular orientation is analysed here in the light of published experiences and research, trying to clarify what it consists of, its keys, its most common practices and why it is valuable for students. Multiperspectivity is at the very core of history as a discipline. It is opposed to memory, characterised by closed stories that impose a single version of the past. In this tension between discipline and memory, the practice of history teaching has usually opted for the latter. But learning to analyse, evaluate and debate the different interpretations of the world of the past (and the present) has much more curricular value. Multiperspectivity not only means understanding the true controversial, open, and critical nature of history, but it is the very foundation of democratic citizenship.
\end{abstract}

Key words: Multiperspectivity. Historical perspectives. Controversial history. Historical thinking. Curricular studies. Secondary School History.

\footnotetext{
* Universidad de Zaragoza, Grupo de investigación ARGOS • ORCID 0000-0003-4958-7239 • jparicio@unizar.es
}

Paricio, J. (2020). El valor de la historia. Estudio de las alternativas curriculares en Secundaria (3): aprender a pensar desde múltiples perspectivas. CLIO. History and History teaching, 46, $202-232$. https://doi.org/10.26754/ojs clio/clio.2020465267. Recibido: 17/3/2020. Aceptado: 10/9/2020. 


\section{Un currículo para aprender a pensar desde múltiples perspectivas}

Hacer creer a los estudiantes un único relato sobre el pasado ha sido la práctica más habitual en la enseñanza de la historia. "La historia tal y como fue", parecemos decirles implícitamente. Pasado e historia se confunden. Se asume y se enseña así que es posible escribir una historia "verdadera". "Si los historiadores, expertos en el currículo y autoridades educativas toman todas las decisiones sobre la versión correcta del pasado, entonces la única tarea de los estudiantes en absorberla. Lo que comenzó como una investigación polémica y debatida sobre la verdad, la valoración y el significado del pasado y el presente, acaba ante los estudiantes como un catecismo que deben memorizar" (Seixas, 2000, p. 21).

Enseñar historia desde múltiples perspectivas significa, en esencia, oponerse a esta visión única del pasado en las aulas. Multiperspectividad, un concepto reciente que ha ido ganando presencia en los debates sobre la enseñanza de la historia desde la década de los 90 (Stradling, 2003), puede definirse como la capacidad de interpretar el pasado desde diferentes perspectivas, entendiendo perspectiva no sólo como una posición historiográfica o ideológica, sino como el conjunto de ideas, creencias, conocimientos, concepciones, intereses, experiencias, emociones o circunstancias contextuales desde la que alguien interpreta y da sentido al mundo del pasado o del presente.

\footnotetext{
"Es necesario que el profesorado cambie la forma tradicional de enseñar historia como un cuerpo establecido de conclusiones sobre el pasado que los estudiantes deben asumir. ... La propia noción de historia como un conjunto establecido de conclusiones distorsiona la naturaleza de la disciplina." (Gibson y Case 2019, p. 254)
}

En el aula de historia, multiperspectividad implica, en primera instancia, incorporar la controversia sobre la interpretación que debe darse a los acontecimientos, acciones o situaciones del pasado. Esto conlleva desarrollar la capacidad de distanciarse de los relatos establecidos y analizar críticamente las diferentes versiones creadas, ya sean fuentes contemporáneas, las narrativas o explicaciones de los historiadores o las interpretaciones de cualquier otro actor social (discursos políticos, productos culturales, ficciones de entretenimiento, etc.). Es un ejercicio de pensamiento crítico y de apertura a perspectivas diferentes y diversas, basadas en contextos, experiencias, 
intereses o puntos de partida distintos. En última instancia, significa profundizar en el modo en que se construyen las historias a partir de los vestigios del pasado y la manera en que esas historias son utilizadas socialmente para modelar nuestro presente.

El procedimiento más habitual es confrontar diversas fuentes o comparar las interpretaciones creadas por diferentes historiadores para un mismo fenómeno, pero podemos pensar en muchas otras formas de introducir esta controversia a través de las lecturas del pasado que podemos encontrar en la literatura, el cine, las imágenes, las tertulias, los discursos políticos... o las propias interpretaciones de los estudiantes. La clave, en cualquier caso, es enseñar que todo hecho puede ser interpretado desde múltiples perspectivas. Se trata, en definitiva, de aprender que no hay discurso humano que no se elabore en el marco de una perspectiva determinada y que es nuestra capacidad para observar, analizar y valorar esa particular perspectiva tras cada discurso la que funda nuestra capacidad de convivencia con la diversidad y nos permite vivir como ciudadanos críticos.

Resulta casi una obviedad afirmar que multiperspectiva y pensamiento crítico van de la mano. Confrontar múltiples perspectivas del pasado exige deconstruir en alguna medida cada discurso, analizando aquello en lo que se funda, su coherencia y sus presupuestos. Analizar críticamente una fuente o un relato histórico significa, en definitiva, distanciarse y elaborar un juicio sobre todo aquello que debe tenerse en cuenta para interpretarlo adecuadamente.

Como en las entregas anteriores de esta serie de artículos (Paricio 2018; 2019), el objetivo global de las líneas que siguen es explorar una determinada forma de orientar el currículo de historia en Secundaria. Por orientación curricular entendemos el propósito fundamental que gobernará todas las decisiones curriculares de la asignatura. Orientar una asignatura de historia hacia la idea de multiperspectiva significa apostar por un determinado "valor" curricular, un tipo de particular de desarrollo del estudiante, como persona y como ciudadano, a través del estudio de la historia. Ese "valor" obligará a seleccionar determinado tipo de problemas, contenidos y metodologías (y dejar fuera muchos otros). Como en ocasiones anteriores, seguiremos una secuencia argumental básica de tres bloques: a) definición precisa de lo que significa esta orientación curricular; b) indicación de algunas ideas y principios 
fundamentales que podrían guiar su puesta en práctica en el aula; y c) argumentación de su "valor" curricular, es decir, aquello valioso que puede aportar al estudiantes estudiar una historia enfocada de este modo.

\section{La idea de multiperspectiva}

La idea multiperspectiva está en el mismo corazón de la historia, como empresa humana. El hecho de que existen múltiples historias sobre un mismo pasado es una obviedad. Basta con asomarse a cualquier librería especializada para comprobarlo. Lo realmente interesante de la idea de multiperspectiva es que proclama que la mayor parte de ellas son "verdaderas" y que ninguna lo es. En otras palabras, lo que el concepto cuestiona es la idea de "verdad" histórica, la suposición de que es posible contar las cosas "tal y como fueron" (o tal y como son). Y esto no sólo es una declaración epistemológica fundamental, sino que encierra un auténtico tesoro educativo.

El termino perspectiva remite a la idea de que lo que uno "ve" depende de la posición desde la que mira. La metáfora óptica tiene algunas limitaciones, pero representa bien una primera idea fundamental: la "posición" desde la que escribe el historiador o la fuente, es decir, sus concepciones, creencias, conocimientos, intereses, cuestiones, valores, contexto o emociones, condiciona de manera directa "su" visión, su historia. Es una idea polémica, pues remite inmediatamente a la tentación de una historia puramente "subjetiva". De hecho, como ha mostrado Vansledright (2002), cuando se intenta demostrar a los estudiantes jóvenes esta naturaleza en perspectiva de la historia, tienden a saltar a una posición de desconfianza absoluta. También Peter Lee (2005) ha recogido testimonios de esta reacción de los estudiantes al descubrir que existen múltiples relatos del mismo pasado: "Todo el mundo tiene derecho a tener sus propias opiniones" (Laurence, $8^{\circ}$ grado) [13-14 años]; "Es una cuestión de opinión" (Briony, $8^{\circ}$ ); "Los historiadores pueden tener muchas opiniones diferentes, así que puede haber diferentes relatos históricos. Sólo uno puede ser verdad, pero no sabemos cuál es" (Xiao Ming, $6^{\circ}$ ). Al descubrir que existen múltiples relatos posibles, la historia se convierte para los estudiantes en una simple cuestión de opinión, cada cual puede pensar lo que quiera. De un salto, parece pasarse de la credulidad absoluta en un relato "verdadero" al relativismo o escepticismo igualmente absolutos. 
Como hemos explicado en otro lado (Paricio, 2021), creer en la "verdad" del relato histórico y pensar que todos ellos no son más que opiniones son dos caras aparentes de una misma posición epistemológica. Lo que llamamos realismo ingenuo no es sino la creencia de que es posible contar la "verdad" sobre los acontecimientos del pasado, las cosas "tal y como fueron". Desde esta perspectiva epistemológica los relatos históricos sólo pueden ser verdad o mentira, correctos o erróneos. Cuando los estudiantes descubren que hay varios relatos propuestos como "verdaderos", simplemente saltan a la siguiente posición: si no son verdad (porque "verdad" solo puede haber una), deben ser mentira.

La clave, por tanto, de la incorporación de múltiples perspectivas al currículo de historia es el aprendizaje de que no sólo existen infinidad de historias válidas posibles, sino que nunca podrá haber una historia que podamos considerar "verdadera", porque no es posible escribir una historia al margen de una perspectiva determinada. No hay, ni habrá nunca, una historia sin perspectiva. Nadie, tampoco ningún historiador, puede despojarse de lo que es para escribir desde fuera de sí mismo, de su particular forma de pensar y de sentir, de sus preguntas, de su contexto o de su manera de entender la historia como disciplina. "Cualquier historia es la historia de alguien" (Levstik, 1997, p. 48). O, como nos recuerda Chapman (2011a), cada historia escrita nos habla tanto del pasado que cuenta, como del historiador que la escribe. Las historias son textos escritos por seres humanos, incapaces de escapar de su propia humanidad, de su particular naturaleza, contexto o tiempo, por mucho que se auto-impongan colectivamente reglas estrictas para escribirlas con rigor y profesionalidad.

\section{Memoria e historia: no hay pensamiento histórico sin múltiples perspectivas}

Peter Seixas (2000; 2007) argumenta que, desde el punto de vista epistemológico y didáctico, hay tres formas de enfrentar el problema de la coexistencia de interpretaciones alternativas del pasado. La primera consiste en buscar y enseñar "la mejor historia", la forma en que "de verdad" ocurrieron las cosas. Esta concepción de la historia está asociada, según Seixas, a la "memoria colectiva". En las aulas, supone obligar a los estudiantes a aprenderse esa "verdad histórica", como un relato fijado por la autoridad, y alejarlos de cualquier indagación o razonamiento histórico. La segunda opción es admitir y presentar a los estudiantes diversas interpretaciones del pasado, 
enseñándoles a enjuiciar cuál puede ser más correcta, en función de las evidencias disponibles o las valoraciones de otros historiadores. La clave es que aprendan los criterios de validez que aplica la disciplina para juzgar los diferentes relatos históricos, lo que lleva a Seixas a llamarla opción "disciplinar". La tercera orientación, "postmoderna", también acepta y presenta diversas versiones del pasado, pero ya no se trata de buscar la versión más válida, sino de explorar cómo cada versión parte de determinadas concepciones, intereses, propósitos o creencias que se plasman en determinadas estrategias narrativas y retóricas.

Este modelo nos servirá de marco fundamental para analizar lo que significa introducir la idea de multiperspectiva en el aula de historia.

Es muy habitual entre los investigadores oponer historia multiperspectiva y memoria histórica (Klein, 2010; Seixas, Fromowitz y Hill, 2005). Frente al relato único del pasado que comparte una comunidad y que le ofrece una conciencia y memoria compartida, la historia disciplinar es investigación crítica, cuestionamiento y apertura continua a nuevas interpretaciones y perspectivas.

La memoria otorga al pasado un único sentido, en el que se fundamenta una determinada visión del presente o un proyecto de futuro. Ya se trate del sentimiento de pertenencia a una nación, del proyecto revolucionario basado en la lucha de clases, de la superioridad moral y política de la democracia o de la confianza en el progreso económico y cultural de la humanidad, la lectura del pasado da soporte a la visión actual. $\mathrm{O}$, dicho en otras palabras, el pasado tiene un sentido... en el presente. Se construye una conciencia histórica que, a través del pasado, da sentido al presente y anticipa el futuro (Rüsen, 2008; 2012). Ya en Tucídides vemos esta idea pragmática de una historia que sea provechosa y sirva para juzgar nuestro presente y futuro. Son relatos, por definición, cerrados, en el sentido de que imponen una estructura de significado sobre los hechos dentro de un determinado marco ideológico y fijan una determinada interpretación que conlleva, habitualmente, un mensaje político o ético (Klein, 2010; Ahonen, 2017). "Siempre que exista un único relato coherente presentado como el relato verdadero estaremos ante un enfoque de la educación histórica orientado a la memoria colectiva" (Seixas, 2007, p. 20). 
Esta concepción de la historia como memoria es predominante en el sistema educativo desde que, en el siglo XIX, "las élites de los estados y la mayoría de los historiadores profesionales dieron por supuesto que una educación en la historia (nacional) era esencial para la 'construcción de la nación' y una 'ciudadanía responsable"” (Berger y Lorenz, 2008, p. 12). La construcción de un "nosotros" que hunde sus raíces en el tiempo y que contagia el sentimiento de pertenencia a un grupo ha sido el eje y propósito fundamental de la enseñanza de la historia. El sujeto de estos relatos cerrados es siempre la propia nación y, a través de ellos, los estudiantes comparten como propios los éxitos y fracasos que han llevado a construir lo que "somos": "para apropiarse de esa identidad, se necesita ser capaz de repetir ese relato histórico, entendiéndolo como algo propio, decir 'nosotros' y 'nuestro'” (VanSledright, 2011) al referirse a ese devenir de la nación en el tiempo.

Por supuesto, esta concepción de la historia como relato único y cerrado es inseparable de la batalla por dominar el sentido del pasado. Ya se trate de la Guerra Civil Española, la "Conquista de América", el Colonialismo, el Holocausto, el imperialismo japonés o los derechos de los nativos en cualquier parte del mundo, las "history wars" (Foster, 1998; Clark, 2006; 2009) en los medios de comunicación o a propósito del currículo de historia se repiten en todos los países del mundo. Dominar el relato del pasado es orientar el sentido del presente y del futuro. "Desde sus comienzos, en sus manifestaciones más primarias y elementales, la historia ha tenido siempre una función social —generalmente la de legitimar el orden establecido-, aunque haya tendido a enmascararla, presentándose con la apariencia de una narración objetiva de acontecimientos concretos" (Fontana, 1999, p. 15). En este sentido, los estudios de Mario Carretero sobre el tratamiento escolar de la presencia española en América son un buen ejemplo de cómo, bajo currículos aparentemente asépticos y objetivos, determinadas visiones e intereses del presente se encarnan en las interpretaciones históricas que los estudiantes aprenden (Carretero, 2001; 2007; 2011b; Carretero y Kriger, 2008: 2011; Carretero y Bermudez, 2012; González y Carretero, 2013).

Frente a esta historia única que nace de una visión cerrada del presente, la idea de una historia abierta al debate entre múltiples perspectivas reivindica tanto la pluralidad democrática y la ciudadanía crítica, como la propia epistemología de la disciplina 
histórica. Este doble corazón epistemológico-disciplinar y político-ciudadano impulsa la práctica totalidad de las propuestas de introducción de la multiperspectiva en el aula de historia.

Aunque la mayor parte de los historiadores profesionales reconocen hoy que en algún punto se diluye la frontera entre memoria e historia disciplinar (Van Nieuwenhuyse y Wils, 2012), existe, a nuestro parecer, una clara distancia entre ambas, marcada por la disposición a considerar y debatir las múltiples perspectivas posibles sobre el pasado y por la exigencia disciplinar de análisis crítico de cualquier historia o fuente. La disciplina histórica es, por definición, multiperspectiva. La conciencia de la particular perspectiva detrás de cada historia y cada fuente es el punto de partida mismo de la disciplina. Por esta razón, debatir con nuestros estudiantes sobre las múltiples perspectivas y versiones posibles de un hecho histórico es, sencillamente, comenzar a enseñarles lo que es la historia como disciplina.

Frente a la primera de las orientaciones descritas por Seixas (memoria) donde se presenta una única perspectiva como "verdadera", la segunda (disciplinar) y la tercera (postmoderna) apuestan por la multiperspectiva y enseñan a los estudiantes a analizar y valorar diversas versiones del pasado. Si en la primera orientación domina el realismo ingenuo, la creencia de que existe una versión verdadera y que es posible contar las cosas tal y como fueron, las otras dos se alejan de esa concepción epistemológica ingenua, en cuanto que admiten que son posibles múltiples historias válidas. Sin embargo, la opción disciplinar al insistir en que los estudiantes aprendan y ejerciten los criterios de validación de la disciplina, les transmite la idea implícita de que existe una "verdad" histórica a la que ajustarse, aunque ninguna historia particular logrará nunca recoger esa verdad por completo. Sólo la tercera orientación recoge verdaderamente, a nuestro juicio, la idea de perspectiva y de multiplicidad de perspectivas en toda su profundidad, en la medida en que asume que la historia es siempre una interpretación del pasado, una construcción de sentido de acuerdo con una determinada perspectiva (Paricio, 2021).

Los requisitos más básicos de cualquier interpretación histórica son, por supuesto, la conformidad con los vestigios del pasado y la coherencia (temporal, lógica, psicológica, sociológica...) del discurso. Una historia no es cualquier "invención" sobre el pasado. Pero esas reglas de juego disciplinares no aseguran la conformidad con 
una pretendida "verdad" del pasado, porque el pasado, en sí mismo, carece de un sentido (verdadero) que hay que descubrir. El pasado tiene tantos sentidos como perspectivas se proyectan sobre ese pasado, porque el sentido no reside en el pasado mismo, sino que lo construyen los seres humanos al interaccionar con ese mundo desde sus desde sus contextos y perspectivas particulares. Somos los seres humanos los que damos forma e inteligibilidad al pasado al representarlo de determinado modo: seleccionando, organizando, relacionando, creando sujetos, enfatizando, valorando...

A nuestro parecer es también esta tercera orientación de Seixas de la multiperspectiva la que contiene un mayor potencial educativo. Indagando sobre las distintas versiones del mundo (pasado o presente), tratando de identificar y analizar las cuestiones, concepciones o creencias que hay detrás de cada una de ellas, recreando los contextos, experiencias, intereses o emociones que las motivan o explorando cómo esas perspectivas se trasladan a determinadas disposiciones, estrategias y retóricas en las representaciones, los estudiantes desarrollan su pensamiento crítico, pero, sobre todo, descubren que no es tanto una cuestión de "verdad" o "mentira", como de tensión y pugna entre representaciones alternativas del mundo. Matizan sus ideas, comprenden la diversidad, se adentran en la complejidad y, sobre todo, comprenden la importancia de los símbolos, representaciones y relatos en el mundo social, su papel en la evolución y la cultura de cualquier sociedad. Los estudiantes aprenden que son las historias que nos contamos sobre nuestro pasado, los relatos que construimos sobre nuestro presente, en definitiva, el sentido que damos al mundo lo que determina nuestras acciones como individuos y como sociedades. Esta es la esencia del concepto de perspectiva o de multiplicidad de perspectivas, el lugar al que llegamos intelectualmente cuando superamos el umbral del realismo ingenuo. Cruzar ese umbral es, en sí mismo, un objetivo curricular de alto nivel, un logro de un valor incalculable, desde el punto de vista personal y social.

\section{Multiperspectiva en los currículos de tres países: entre la ciudadanía democrática y el pensamiento histórico}

La idea de multiperspectiva puede conformar el currículo de historia de formas muy diversas. Reseñamos a continuación tres ejemplos muy diferentes de hacerlo a través de los currículos de Australia, Suecia y Alberta (Canada). Los dos primeros casos son 
currículos que podríamos llamar "disciplinares", en los que el propósito es el desarrollo de la capacidad de pensamiento histórico, aunque con orientaciones distintas. El tercero, el currículo del estado de Alberta, está orientado a la formación de una ciudadanía capaz de convivir con la pluralidad y la controversia. En los tres casos, la multiperspectividad constituye el eje nuclear del currículo.

El texto oficial del currículo australiano de historia (ACARA, 2015) coloca "perspectives" como uno de los siete conceptos clave de la comprensión histórica que articulan todo el currículo. Entiende este concepto en un doble sentido: por un lado, la perspectiva desde la que actuaron las personas del pasado, lo que habitualmente llamamos empatía o toma de perspectiva histórica, aunque poniendo el énfasis en las múltiples perspectivas que ya coexistían en ese momento del pasado: "no todas las personas de un sociedad determinada compartían la misma perspectiva. Como hoy, podía haber diferencias dramáticas en los valores, actitudes y prácticas entre las personas de una misma sociedad del pasado, lo que producía inestabilidad, conflicto y agitación. Estudiar esas diferencias de perspectiva en un momento histórico, y el conflicto subsiguiente, puede ayudar a los estudiantes a comprender las raíces del conflicto en su propio mundo y ofrecerles claves para la posible resolución de ese conflicto" (s.p.). Por otro lado, el currículo australiano describe un segundo significado de perspectives, concebido ahora como las perspectivas, muchas veces discrepantes, desde las que los historiadores o la gente en general construye sus relatos $e$ interpretaciones del pasado y propone que los estudiantes profundicen en el origen de esas discrepancias. Un doble enfoque de la multiperspectiva que representa lo que podríamos llamar un plantemiento clásico de la idea de "múltiples perspectivas" en el currículo.

El currículo sueco (Swedish National Agency for Education, 2018) es, a nuestro juicio, uno de los más interesantes del mundo en su apuesta por la comprensión de la naturaleza en perspectiva del discurso histórico y sus usos sociales. Su eje es la conciencia histórica, aunque en un sentido crítico muy alejado de la tradicional absorción de narrativas cerradas identitarias. Muy al contrario, lo que pretende es que los estudiantes sean muy conscientes del papel social que juegan esas narrativas históricas y cómo modelan nuestro presente, en la línea defendida por autores como Rüsen (2004) o Duquette (2015): 
"La comprensión de los seres humanos del pasado se entrelaza con sus creencias sobre el presente y sus perspectivas de futuro. De esta forma, el pasado afecta a nuestras vidas hoy y nuestras elecciones para el futuro. Mujeres y hombres de todas las épocas han creado narrativas históricas para interpretar la realidad y conformar su propio contexto. (...) La enseñanza debería contribuir a que los estudiantes desarrollasen su comprensión de cómo las narrativas históricas son utilizadas en la sociedad y en la vida cotidiana. De esta forma, los estudiantes deberían desarrollar diferentes perspectivas sobre sus propias identidades, valores, creencias y las de otros" (p. 208).

El currículo sueco recoge así ese enfoque "postmoderno" de la enseñanza de la historia del que hablaba Seixas (2000) en el que se cuestiona la propia conciencia histórica a través de la multiperspectiva. Lo que se busca, en síntesis, es que los estudiantes: a) reconozcan el carácter construido (no natural) de la conciencia histórica y la identidad de los grupos; b) sean conscientes del modo en que determinadas narrativas constuyen esa conciencia histórica; y c) aprendan a analizar las perspectivas (intereses, concepciones, creencias...) que modelan esas narrativas.

La capacidad de análisis crítico de estas narrativas pasa, obviamente, por "desarrollar la capacidad de plantear cuestiones y evaluar las fuentes como base del conocimiento histórico" y "examinar de forma crítica, interpretar y evaluar" esas fuentes (p. 208). Esto les permitirá "reflexionar sobre cómo ellos mismos y otros hacen uso de la historia en diferentes contextos y desde diferentes perspectivas" (p. 209).

El currículo de Ciencias Sociales del estado canadiense de Alberta (Alberta Ministry of Education, 2015) constituye un ejemplo bien diferente de una orientación hacia la formación de esa capacidad de afrontar múltiples perspectivas. En este caso, el foco principal está puesto en desarrollar la competencia de convivir en "la realidad plural de la sociedad canadiense". Uno de los objetivos fundamentales es "valorar y respetar las múltiples perspectivas que conforman la realidad política, socioeconómica, lingüística y cultural de Canada, incluyendo la perspectiva de las culturas aborigen y francófona" ( $p$. 2). En este currículo orientado a formar una ciudadanía capaz de "construir una sociedad que es plural, bilingüe, multicultural y democrática” (p. 1), un enfoque en multiperspectiva de la historia y la geografía se ofrece como una vía "para comprender cómo la cohesión social puede lograrse en una sociedad plural” (p. 2).

Los términos "pluralidad", "diversidad", "multiples perspectivas" y "multiculturalidad" se repiten por todo el texto de referencia, dejando claro que el estudio de la historia debe 
contribuir a hacer posible la convivencia en una sociedad marcada por los enfrentamientos y reclamaciones entre las tres comunidades: "estudiar las cuestiones controvertidas es importante para preparar a los estudiantes para participar de forma responsable en una sociedad democrática y plural. Esta materia debe representar la oportunidad de desarrollar la capacidad de pensar con claridad, razonar lógicamente, examinar los diferentes puntos de vista con mentalidad abierta y respeto y formular valoraciones fundamentadas" (p. 6).

Las orientaciones curriculares de estos tres países ilustran ese doble corazón que impulsa la idea multiperspectiva en la enseñanza de la historia: por un lado, el desarrollo de una ciudadanía democrática, entendida como capacidad de afrontar racionalmente la diversidad, la controversia e incluso el conflicto. Por otro lado, la comprensión de la naturaleza de la historia, siempre dependiente de la perspectiva desde la que se construye y siempre sujeta al debate, tanto académico como social. Sin la comprensión de esta naturaleza multiperspectiva, crítica y polémica resulta imposible avanzar en el desarrollo del razonamiento histórico, como ya demostraron los estudios del proyecto CHATA (Lee, 1998; Lee y Ashby, 2000; Lee y Shemilt, 2003; 2004) o los de Arthur Chapman (2011b; 2011c), y tampoco es posible comprender la controversia sobre los usos que la sociedad hace de la historia. Y sin la capacidad de analizar y comprender las múltiples perspectivas y narrativas que se entrecruzan y confrontan en nuestra sociedad, resulta difícil mantener la idea ilustrada de una sociedad "razonable" (Lipman y Sharp, 2002), capaz de dialogar sobre sus distintos puntos de vista y canalizar sus conflictos.

\section{La práctica de una educación histórica basada en la multiperspectiva}

Un currículo que coloque como uno de sus ejes curriculares la idea de multiperspectiva es, por definición, un currículo problematizado en el que los estudiantes afrontan una serie de problemas relacionados con la interpretación histórica de determinados sucesos, acciones o situaciones. Los estudiantes se enfrentan al análisis y valoración de diferentes versiones e interpretaciones de los hechos, que pueden provenir de distintas fuentes, diversos historiadores o de cualquier otra persona, del pasado o del presente. 
Siguiendo el esquema de Peter Seixas (2000), el estudiante puede realizar dos tipos de tares con esas interpretaciones diversas del mundo del pasado. Puede, dentro del enfoque disciplinar, revisar las fuentes que sostienen cada una de las versiones, tratando de decidir cuál de ellas está mejor fundamentada; y/o puede, por otro lado, analizar textos y contextos, tratando de identificar y describir las perspectivas que hay detrás de cada uno de ellos. Son dos ejercicios de naturaleza muy diferente que, por supuesto, pueden complementarse entre sí.

El enfoque disciplinar del modelo de Seixas, requiere introducirse en el corazón del método del historiador. Peter Lee defiende este enfoque señalando que "sin una reflexión y enseñanza explícita sobre la naturaleza de las evidencias históricas y de los discursos históricos, así como de las distintas formas en que se puede comprobar la validez de una afirmación, las perspectivas múltiples se convierten en simplemente una nueva razón para no tomarse en serio la historia" (2005, p. 70). Ante el riesgo cierto, como hemos visto, de que la disciplina histórica sea tomada por mera opinión o se desdibujen las fronteras con la literatura, los estudiantes aprenden el rigor del método histórico, aquello que diferencia la historia profesional de los relatos ordinarios sobre el pasado. Los estudiantes descubrirán así que no es posible comprobar directamente ni siquiera los "hechos" más básicos y que todo es, a menudo, un inmenso puzzle en el que fuentes muy diversas van dando informaciones parciales y a veces poco concluyentes. Como "detectives", una metáfora extensamente utilizada en este tipo de actividades en el aula (Wineburg, 2010), se supone que los estudiantes deben aprender a interrogar los restos y vestigios del pasado para ir encajando las piezas de los hechos, como lo hacen los historiadores.

Por nuestra parte, no estamos seguros de que tal propuesta no plantee grandes dificultades para poder ser llevada al aula. Evaluar la validez de distintas interpretaciones históricas a partir del análisis de las fuentes que las sustentan requiere, no sólo un conocimiento muy profundo de la época, sino unas capacidades de indagación disciplinar verdaderamente profesionales, que creemos que están muy lejos del alcance de la mayoría de los estudiantes de Secundaria o Bachillerato. Los estudiantes pueden "jugar" (en el mejor sentido) a ser detectives a partir de algunas evidencias, como método para comprender por dónde se comienza a escribir la historia, pero difícilmente pueden juzgar la validez de un relato histórico creado por un 
profesional. El estudio publicado por Wineburg $(1991 ; 2001)$, en el que se dio a ocho historiadores y a un grupo de estudiantes unas serie de documentos sobre la Batalla de Lexington, mostró de modo muy concluyente la distancia enorme que separa el razonamiento de unos y otros a partir de las mismas fuentes, incluso en el caso de historiadores no especialistas en esa época. Así que, a nuestro juicio, la pregunta es ¿resulta realista pensar en formar a los estudiantes en la interpretación de fuentes históricas hasta alcanzar el nivel mínimo requerido para poder juzgar la validez de unas y otras interpretaciones del pasado?, ¿qué cantidad de documentos deberían revisar como para comprobar la validez de, digamos, dos interpretaciones distintas de la "Conquista de América" o de la Guerra Civil?

Mucho más interesante y realista resulta, a nuestro juicio, la alternativa que Seixas llama enfoque postmoderno. La idea básica es que los estudiantes se enfrenten a diversas interpretaciones y, con la ayuda de información u otras fuentes, extraigan conclusiones sobre las perspectivas que animan las distintas versiones del pasado. Se puede comenzar por ejercicios muy básicos, como comparar varios relatos históricos y observar allí lo que cada uno de ellos incluye y lo que deja fuera. Se trata de aprender a distanciarse de las interpretaciones y la comparación entre versiones ayuda a percibir que cada una de ellas se centra en aspectos diferentes y que puede estar buscando dar respuesta a cuestiones diversas o persiguiendo intereses distintos. Es posible también aprender a observar las valoraciones implícitas que están detrás del uso de determinadas expresiones o cómo determinadas disposiciones narrativas sugieren o llevan al lector a determinados tipos de interpretación. Pueden debatir sobre todo ello, extrayendo conclusiones importantes sobre el modo en que, sin falsear realmente los hechos básicos, poniendo el foco en aspectos particulares o disponiendo el discurso de determinado modo, es posible construir historias muy diferentes e incluso opuestas.

Con un soporte adecuado, pueden dar un paso más y explorar las concepciones, creencias o experiencias que puede haber detrás de determinados discursos históricos. Este tipo de trabajo requiere habitualmente materiales adicionales (datos, fuentes, información...), a través de los cuáles los estudiantes pueden explorar el contexto, en particular, cuando se trata de fuentes primarias sobre una época determinada. Esta contextualización es la primera y una de las más importantes vías 
para la reconstrucción de la perspectiva desde la que se conforma cualquier fuente escrita o realización material. El análisis de las múltiples perspectivas desde las que diferentes personas o medios de comunicación describían e interpretaban la Guerra del Rif a principios del siglo XX, por ejemplo, requiere que los estudiantes exploren el contexto social, político, económico y cultural de la época, analizando las tensiones y conflictos entre diferentes grupos sociales, recreando las distintas ideas, concepciones y experiencias desde las que cada parte en conflicto interpretaba la situación y rastreando como esas ideas se plasmaron en discursos con lenguajes muy diferentes que parecen describir mundos distintos. Los estudiantes no sólo exprimen así las fuentes para aprender en profundidad algunas claves de la época, sino que aprenden a tomar distancia crítica sobre los discursos y descubren cómo distintas experiencias y contextos modelan ideas y concepciones muy diferentes sobre el mundo, que se plasman en discursos alternativos o en conflictos abiertos.

Las múltiples perspectivas que los estudiantes exploran pueden ser de naturaleza muy diferente. Puede tratarse de fuentes primarias con versiones alternativas más o menos contemporáneas de la época estudiada, de las diferentes lecturas que en momentos históricos distintos se ha ido haciendo de determinado fenómeno histórico, de las distintas historias escritas por historiadores profesionales actuales o de las lecturas del pasado que hacen en la actualidad distintos agentes o grupos sociales actuales. Se pueden investigar las versiones confrontadas que provocan los conflictos sociales o se puede indagar en problemas historiográficos abiertos a través de las historias escritas por distintos historiadores. También se pueden traer al aula las versiones muy diferentes y mucho más íntimas que nos dan las personas que han vivido los acontecimientos directamente y que dependen de las experiencias concretas que vivieron o de su situación particular, o podemos explorar la visión que en la cultura popular modelan el cine, los documentales, la literatura o los videojuegos, contrastándolas entre sí (particularmente si son de épocas distintas) o comparándolas con versiones de los historiadores.

Las actividades pueden ser muy diversas, pero todas ellas comparten cuatro aspectos: a) afrontar las múltiples perspectivas exige a los estudiantes razonar históricamente sobre determinados momentos y aspectos del pasado, condición indispensable para una comprensión profunda de la historia (Vansledright, 2011); b) la exploración de 
esas perspectivas diversas conlleva un acercamiento a la complejidad de las sociedades de cualquier época, un objetivo de aprendizaje fundamental en las ciencias sociales; c) la deconstrucción de los distintos discursos lleva implícito un aprendizaje sobre el modo en que las diferentes perspectivas se proyectan en representaciones y símbolos $y$, a su vez, esas representaciones nos modelan como individuos y sociedades; y d) la comparación y análisis crítico de múltiples perspectivas es la vía más inmediata para la superación del realismo ingenuo y la comprensión de la naturaleza multiperspectiva de la historia. Estos cuatro aspectos -comprensión profunda de los hechos del pasado, acercamiento a la complejidad social, importancia de la interacción simbólica y comprensión de la naturaleza del razonamiento históricoson cuatro "valores" de altísimo nivel que cualquier actividad relacionada con la multiperspectividad debería potenciar de manera particular.

\section{Los temas que se trabajan en las actividades de multiperspectiva}

La introducción de la multiperspectiva en el currículo de historia ha estado ligada muy habitualmente al tratamiento en el aula de temas controvertidos en los que convergen versiones confrontadas, a veces de forma abrupta y muy polarizada. En ocasiones, incluso forma parte de las políticas de reconciliación en escenarios sociales posteriores a conflictos abiertos (e.g. Ahonen, 2012; Korostelina y Lässig, 2013; Elmersjö, Clark y Vinterek, 2017). Desde la "Conquista de América" (Bermudez y Argumero, 2018), la colonización holandesa en Indonesia (Mark, 2017), la historia de la esclavitud en los Países Bajos (Keet y Datema-Chang, 2020) o la colonización y derechos de los nativos del Canada (Seixas, 2000; Scott, 2013; Gibson y Case, 2019), hasta las propuestas de Stuart Foster sobre la interpretación y conmemoración de la Primera Guerra Mundial (Foster y Roch, 1997; Foster, 2017), el conflicto en Irlanda del Norte (Barton y McCully, 2007; 2012), la Guerra Civil finlandesa (Ahonen, 2012; Hakoköngäs, Kleemola, Sakki y Kivioja, 2020), la división de la Finlandia de después de la Segunda Guerra Mundial (Ahonen, 2019), la resistencia, acomodación o colaboración de los holandeses en la persecución de los judíos durante la ocupación alemana (Kropman, van Drie y van Boxtel, 2019) o el conflicto racial en South Africa (Ahonen, 2012), todas ellas son situaciones históricas cuya interpretación es controvertida en el presente desde el punto de vista ético y político y para cuyo tratamiento en el aula se propone un enfoque multiperspectivo. 
Sirkka Ahonen, en su estudio comparado del uso de la historia en tres sociedades recién salidas de un conflicto abierto (Finlandia, South-Africa, Bosnia-Herzegovina), describe con precisión cómo se modela la memoria colectiva, prolongando el conflicto:

\begin{abstract}
"En la memoria pública y en los materiales educativos, la atribución mutua de culpabilidad se repite en las tres sociedades. "Nosotros" fuimos víctimas de atrocidades y traiciones tanto como lo fueron "ellos" en su memoria. "Ellos" fueron culpables de las mismas ofensas que "nosotros" lo somos en sus acusaciones. Todas las partes utilizan mitos socializados para asentar sus afirmaciones de superioridad moral (2013, p. 94).
\end{abstract}

La argumentación más tradicional defiende que la multiperspectividad puede ayudar a los estudiantes a romper con este atrincheramiento en los mitos de culpabilización y victimización que mantienen vivo el antagonismo. En estas actividades se anima a los estudiantes a reconocer y comprender los valores y perspectivas que animan las distintas facciones en conflicto (Keet y Datema-Chang, 2020). Mediante el análisis de los contextos y experiencias de diversos actores, los estudiantes aprenden a mirar las situaciones desde diferentes puntos de vista y desarrollan empatía por las diferentes partes en conflicto (Stradling, 2003).

Sin embargo, el tratamiento de los temas controvertidos en el aula no es tan sencillo como analizar racionalmente las diferentes perspectivas. Keet y Datema-Chang (2020) advierten sobre las emociones implicadas en estos temas controvertidos y cómo es necesario utilizar esas emociones y no dejar que bloqueen el proceso de aprendizaje. En este mismo sentido, el estudio de Barton y McCully (2007) sobre el conflicto en Irlanda del Norte cuestiona la idea generalizada de que basta con establecer una discusión racional para aprender a afrontar la controversia. Según estos autores, ignorar las emociones puede llevar a que simplemente los estudiantes consideren irrelevante la discusión:

\footnotetext{
"Las entrevistas con el profesorado más experimentado sugieren que esto les requiere 'contener sus nervios' cuando los estudiantes responden de manera muy emocional. Particularmente al comienzo de las discusiones $-y$ especialmente en el caso de estudiantes que no han tratado estos temas en la escuela antes- el profesorado puede necesitar permitir una discusión más altamente cargada de lo que se consideraría permisible en otros contextos. Reducir la discusión demasiado rápido porque parece irse de las manos es tentador, pero las reacciones fuertes -e incluso irracionales- pueden ser necesarias como un primer paso antes de que los estudiantes aprendan a expresarse de forma más articulada y respetuosa. Puede ser también necesario facilitar que una sesión altamente emocional vaya reduciéndose progresivamente en vez de cortarla abruptamente cuando se acaba el tiempo" (p. 15)
} 
Por otro lado, en estos casos controvertidos, no se trata únicamente de conocer dos versiones enfrentadas, sino de ampliar todo lo posible las perspectivas introduciendo matices y experiencias muy diversas que permitan a los estudiantes superar una visión en bandos opuestos. Barton y McCully (2007) hablan de "activar la diversidad" para hacer ver a los estudiantes que cada grupo no es homogéneo y que esconde en su interior vivencias y posiciones muy diferentes. En el mismo sentido se pronuncian Kropman, van Drie y van Boxtel (2019), quienes describen la introducción de perspectivas más personales, con contextos particulares, como la forma de hacer posible que los estudiantes exploren esa complejidad inherente a cualquier acontecimiento, frecuentemente barrida por las grandes interpretaciones en conflicto y sus dogmáticos mensajes políticos o éticos.

Pero la multiperspectiva no es un dominio exclusivo del tratamiento de estos grandes temas conflictivos. La idea de multiperspectividad también puede aplicarse al tratamiento de problemas historiográficos clásicos, de manera que los estudiantes se adentren en esa naturaleza interpretativa de la historia como disciplina. Por ejemplo, Nygren, Vinterek, Thorp y Taylor (2017) trabajan con estudiantes de entre 14 y 16 años de Alemania, Inglaterra y Suecia diferentes historias sobre las causas de la Primera Guerra Mundial. Los estudiantes recibieron copias traducidas de artículos escritos sobre el tema por cuatro historiadores y, organizados por grupos, debían convencer a los otros grupos de que su artículo ofrecía las mejores explicaciones de las causas de la Gran Guerra. Tras este ejercicio, los estudiantes recibían un texto con la explicación tradicional y popular que se da en sus respectivos países: "esta lectura crítica final fue diseñada para permitir a cada estudiante problematizar su propia representación a la luz de las diversas perspectivas introducidas durante el debate" ( $p$. 211). Abdou (2017), por su parte, explora nuevas perspectivas historiográficas en el tratamiento de de las civilizaciones de la Antigüedad que vayan más allá de la tradicional focalización eurocéntrica en Grecia y Roma y la idea de una cultura dominante que se difunde a otros pueblos. Propone llevar al aula otras culturas, así como visiones más equilibradas de los intercambios materiales y no materiales entre las diversas civilizaciones y el aprendizaje recíproco que se produce entre ellas. Estas visiones son concebidas como contra-narrativas frente a las narrativas dominantes en los libros de texto. Kropman, van Boxtel y van Drie, (2020), como un tercer ejemplo, estudian las distintas versiones que estudiantes holandeses y flamencos trabajan a 
través de libros de texto sobre la revuelta e independencia de Holanda frente a la corona de Felipe II. Este estudio, como el anterior de estos mismos autores sobre la Holanda ocupada de la II Guerra Mundial (Kropman, van Boxtel y van Drie, 2019), muestra cómo es posible analizar las disposiciones narrativas y metáforas utilizadas en los relatos del pasado, los libros de texto en este caso, como punto de partida de la reflexión sobre las perspectivas implícitas desde las que se escriben.

Otras actividades buscan traer a los estudiantes las voces más personales de las experiencias de las personas del pasado. Por ejemplo, David Waters (2014) propone utilizar un antiguo edificio de Londres en el que residieron familias inmigrantes de hugonotes franceses, así como familias irlandesas, polacas y rusas llegadas a Londres, para que los estudiantes construyan un fresco de múltiples narrativas con las experiencias y las perspectivas de personas muy diversas. "La misma fábrica del edificio, las muchas historias que sugieren en sus distintas capas, disparan la imaginación", porque "los edificios, como los lugares, pueden tener múltiples identidades" (pp. 42-43). Utilizando fuentes contemporáneas de los diversos habitantes de la casa en los siglos XVIII y XIX, los estudiantes indagan sobre sus vidas y la vida del barrio en general en la época, su diversidad como zona de inmigrantes y los sucesivos cambios en el uso del edificio, conforme iba cambiando Londres y el mundo en general.

Menos numerosas son las publicaciones que estudian el trabajo de los estudiantes con las versiones del pasado contenidas en los medios y debates públicos. Heather Sharp (2017) propone a estudiantes de Bachillerato analizar las diferentes versiones que jalonan el agrio debate público sobre la interpretación de la implicación australiana en la Primera Guerra Mundial y, en particular en la campaña de Gallipoli. Los estudiantes eran invitados a responder a una serie de cuestiones a partir de fuentes muy diversas que incluían, entre otras cosas, fotografías, la sinopsis de una miniserie de televisión, el diario de un soldado, o testimonios de las ceremonias de conmemoración. El objetivo fundamental era "invitarles al análisis crítico de los mensaje que han recibido de esas fuentes" (p. 240) y provocar una reflexión sobre los puntos de vista que deberían, a su juicio, ser tenidos en cuenta en el relato público de Gallipoli. Schnakenberg (2010) propone trabajar las caricaturas y dibujos satíricos de la prensa de la época para el estudio de las múltiples interpretaciones de los procesos que 
dieron fin a la I y II Guerra Mundial en Alemania. Elige estas formas de representación porque, casi por definición, representan interpretaciones controvertidas y se ofrecen como acertijos que están pidiendo ser descifrados, aportando un alto nivel potencial de motivación. Por su parte, Marcus y Stoddard (2009) proponen trabajar las diversas perspectivas contenidas en los documentales históricos. Bien analizando la perspectiva general de cada uno de los documentales, o bien aprovechando la diversidad de testimonios que algunos de ellos pueden contener, los autores defienden la particular efectividad de trabajar este tipo de documentos para desarrollar la capacidad de los estudiantes para deconstruir críticamente los discursos, habida cuenta de que los estudios muestran que tienden a ser muy poco críticos con los productos audiovisuales y asumirlos de forma casi inconsciente como verdades.

\section{El valor de un enfoque de la historia en multiperspectiva}

¿Qué aporta de valor para el estudiante trabajar múltiples perspectivas de la historia? Hemos sintetizado los argumentos en siete puntos:

\section{Entendimiento y superación del conflicto y los prejuicios}

El Consejo de Europa (2002) incluye la multiperspectiva como uno de los ocho objetivos de la historia para el siglo XXI: "hacer posible que los estudiantes desarrollen la capacidad para analizar e interpretar la información de forma crítica y responsable, a través del diálogo, a través de la búsqueda de evidencias históricas y a través del debate abierto basado en la multiperspectividad, especialmente sobre cuestiones sensibles y controvertidas" (p. 5). Se busca reforzar la confianza y la comprensión mutua entre los pueblos, eliminando prejuicios y saliendo al paso de la "falsificación y manipulación de la historia" con fines ideológicos. El mandato de introducir múltiples perspectivas para abordar los puntos sensibles o conflictivos entre países apunta al primero y quizás más estudiado de los valores potenciales de la multiperspectividad en el aula de historia: contribuir a salir de las trincheras de los relatos cerrados en los que se exalta el "nosotros" y se maligniza o culpabiliza al "otro", como ha descrito Sirkka Ahonen (2012). 


\section{Valores éticos}

El vínculo entre valores éticos y aprendizaje de la historia ha sido defendido desde la Antigüedad: "el conocimiento histórico que no es guiado por una orientación moral o por juicios morales es históricamente inútil: porque, cómo podríamos comprometernos con un proyecto histórico para todos si no podemos nosotros mismos otorgarle una orientación moral" (Seixas, 2005, p. 144). La solución habitual es exponer a los estudiantes determinadas narrativas de episodios históricos que podríamos calificar de ejemplares -en sentido positivo o negativo- para que asuman sus valores implícitos. Sin embargo, la introducción de múltiples perspectivas puede proporcionar una vía mucho más crítica de acercamiento a los valores. Jaap Schuitema (2008) propone debatir valores a través de múltiples perspectivas sobre situaciones históricas éticamente conflictivas. Se espera de los estudiantes que analicen en profundidad las diferentes perspectivas y contextos en los que se anclan los diferentes valores en conflicto y que elaboren su propio punto de vista considerando esas múltiples perspectivas. El autor subraya como este enfoque no afecta negativamente al nivel de conocimientos adquiridos, sino que, al contrario, "puede hacer esta materia más significativa para los estudiantes y probablemente ayudarles a una comprensión más profunda del tema. Implementando de este modo la educación ciudadana como parte integral del currículo, se puede hacer una contribución valiosa a la educación de los estudiantes" (p. 89).

Actividades de este tipo requieren, tanto de estudiantes como de profesorado, desarrollar una posición de apertura y de revisión crítica de las propias perspectivas y estereotipos culturales. Es necesario situarse en la comprensión de que nuestros principios y normas de comportamiento están contextual e históricamente determinados. Un buen ejemplo lo constituye la actividad que "Nell", la profesora del estudio de Kropman, van Drie y van Boxtel (2019), propone a sus estudiantes sobre la persecución de los judíos durante la ocupación de Holanda por los alemanes. Nell introduce no sólo el clásico ejemplo moral del profesor Cleveringa, que alzó la voz desde su clase frente a las acciones nazis contra los judíos holandeses, frente a una mayoría de holandeses que callaba u otro grupo que colaboraba, sino que trae al aula también otras perspectivas que hacen más compleja la interpretación ética: desde un fragmento de una novela autobiográfica de un hijo de un miembro del partido nazi holandés, hasta un debate sobre la visión de los descendientes de los ocupantes 
alemanes que sisten a la conmemoración anual de la liberación holandesa. Frente al relato cerrado tradicional de "buenos" y "malos" (resistencia/colaboracionismo), la profesora introduce así otras voces y perspectivas que llevan a los estudiantes a debatir y matizar sus posiciones.

\section{Competencias interculturales}

Nordgren y Johansson (2015) asocian la introducción de múltiples perspectivas en el aula de historia con el desarrollo de la competencia intercultural de los estudiantes. Según estos autores la multiperspectiva contribuye a "la capacidad de ponerse en otra posición, interpretar representaciones con las que no se está familiarizado y mediar entre perspectivas" (p. 7). Estas capacidades se asocian al descentramiento de los estudiantes, es decir, su capacidad de relativizar y cuestionar los propios valores y creencias y "ser capaz de ver cómo podrían ser las cosas desde la perspectiva de otro que tiene diferentes valores, creencias y comportamientos" (p. 7). Esto pasa, según los autores, por abrir las narrativas históricas a otras perspectivas y voces de diferentes culturas y considerar, por ejemplo, la representación que actores no occidentales, tradicionalmente ocultos o pasivos, hacen de grandes hechos históricos como la Segunda Guerra Mundial o las Cruzadas. En el mismo sentido, Maria Grever (2012), identificando multiperspectiva y pluralidad, enfatiza la necesidad de que los estudiantes aprendan a convivir con la diversidad propia de las sociedades de nuestro tiempo y a interpretar el mundo integrando discursos plurales: "como cualquier otra persona, estos estudiantes tratan de interpretarse a sí mismos y el mundo que les rodea adaptando e incorporando discursos de sus familias, profesores, compañeros, internet y otras fuentes e integrando todas ellas en una narrativa coherente" (p. 77). En definitiva, no se trata sólo de aprender en la multiperspectiva a vivir sin la seguridad y reafirmación que produce la homogeneidad social y los discursos identitarios cerrados, sino también de aprender a escuchar esos discursos alejados de nuestros propios puntos de vista que provienen de grupos con culturas diferentes y aprovechar ese extrañamiento para cuestionar nuestras perspectivas y valores y construir narrativas abiertas e integradoras.

\section{Comprender la complejidad social}

Al analizar múltiples perspectivas sobre los hechos, los estudiantes aprenden también a afrontar la complejidad y la incertidumbre propia del mundo social, una competencia 
de gran valor en el mundo contemporáneo (Barnett, 2000; 2007). Los estudiantes parten de una visión simplista y reduccionista del funcionamiento social, tal y como han demostrado los estudios sobre el aprendizaje de la causalidad histórica (Pozo y Carretero, 1989; Stoel, van Drie y van Boxtel 2015; Alcoe, 2015), de la empatía histórica (Lee y Ashby, 2001; Brooks 2009; Huijgen, van Boxtel, van de Grift, Holthuis, 2016), del cambio y la continuidad (Lee, 2005; Fordham, 2012) o los conceptos históricos de naturaleza social (Carretero, 2011a; Carretero y Lee, 2014). Sintetizando mucho, podemos decir que tienden a concebir como simple lo que es complejo, como estático lo que es dinámico, como concreto e individual lo que es abstracto o social, o tomando como algo dado lo que puede ser problemático o incierto. Afrontar múltiples perspectivas les obliga a revisar varios de estos aspectos, particularmente el primero y el último. Los estudiantes simplifican cada periodo del pasado atribuyéndole unas determinadas características de manera uniforme. Analizar diferentes voces de la época les hace observar que las tensiones y diferencias son una constante en las sociedades humanas y que siempre hay muchas formas distintas de ver las cosas. Por otro lado, les enseña a situarse en la incertidumbre que es propia de la comprensión de lo social, no dando ninguna versión por verdadera, sino navegando críticamente entre todas ellas.

\section{La batalla social por la hegemonía simbólica}

Por otro lado, analizar los relatos diversos sobre una misma realidad que, en la actualidad o en épocas pasadas, defienden distintos actores o grupos sociales desde diferentes concepciones, intereses o creencias, pone ante los ojos de los estudiantes la batalla social por la hegemonía simbólica. Desde nuestro punto de vista, este es un resultado de muy alto nivel y relevancia. Comprender el papel que los relatos y símbolos compartidos juegan en la vida de las comunidades y cómo la tensión o la lucha social se plasma en relatos y símbolos alternativos, significa para los estudiantes dar un gran salto adelante en la comprensión de los mecanismos sociales.

El aprendizaje de la historia no puede ser ajeno a la cuestión de los usos sociales de la historia. Ya sea para construcción de la identidad colectiva, la defensa de valores, derechos o libertades o el señalamiento del enemigo, la historia es utilizada para movilizarnos en el presente. Las llamadas history wars que inundan los medios en todos los países son el testimonio más evidente de esa lucha por el dominio de 
discurso social sobre el pasado. ¿Puede el aula de historia quedarse al margen de esta batalla por la dominación simbólica de la sociedad?, ¿debería escabullir su responsabilidad de preparar a los estudiantes para situarse en la polémica de forma razonada y consciente? Enseñar a analizar las visiones e intereses que hay, inevitablemente, detrás de las diversas versiones del pasado es la única vía honesta de tratar la dimensión social y política de la historia.

\section{Pensamiento crítico}

Multiperspectiva y pensamiento crítico son dos caras de la misma moneda. "La gran mayoría de las personas no han decidido libremente qué creer, sino que, por contra, han sido socialmente condicionadas (adoctrinadas) en sus creencias", señalan Richard Paul y Linda Elder (2008, p. 3) para justificar la necesidad del pensamiento crítico en el currículo. Para estos autores el pensamiento de las personas sin pensamiento crítico está basado en prejuicios, estereotipos y sobresimplificaciones y sometido "a fuerzas personales y sociales que ni controlan ni comprenden y que no obedecen a sus propios intereses" (p. 3).

El pensamiento crítico está en el mismo centro del razonamiento histórico. Formar un juicio crítico sobre cómo interpretar las fuentes o los relatos históricos a través del análisis de su fiabilidad, consistencia y perspectiva es la base del trabajo del historiador. Y muy probablemente, la introducción de múltiples perspectiva en el aula de historia sea una de las formas más efectivas de desarrollar el pensamiento crítico de los estudiantes. Enfrentarse a multiplicidad de versiones ofrece la oportunidad de observar y valorar la consistencia y perspectiva de cada versión, ya se trate de fuentes, historias profesionales o relatos en los medios sociales. Tenemos todavía pocos estudios que confirmen esta hipótesis, pero, por ejemplo, los resultados de la experiencia de Nygren, Vinterek, Thorp y Taylor (2017) sobre las múltiples versiones de las causas de la Primera Guerra Mundial muestran que, efectivamente, conforme los estudiantes incrementan su capacidad para contemplar diversas perspectivas sobre una realidad, aumenta su capacidad para realizar un análisis crítico de los relatos que circulan tradicionalmente en su medio social. 


\section{El descubrimiento de la naturaleza de la historia y el razonamiento histórico}

Por último, confrontar los relatos y explicaciones del pasado que construyen los distintos historiadores enseña a los estudiantes la verdadera naturaleza de la historia como disciplina, su carácter necesariamente interpretativo y polémico, el modo en que las diversas perspectivas crean historias diferentes, incluso desde criterios de validación compartidos. Se trata de hacer evolucionar sus concepciones epistemológicas ingenuas: la creencia de que es posible representar el pasado (o el presente) como en un espejo, "tal y como es", es decir, lo que llamamos realismo ingenuo. Este cambio conceptual epistemológico es fundamental no sólo para cualquier forma de razonamiento histórico, como demostraron los trabajos del proyecto CHATA (Lee, 1998; Lee y Ashby, 2000; Lee y Shemilt, 2004), sino que tiene un gran valor personal y social (Paricio, 2021).

En definitiva, apostar por introducir la multiperspectiva en el currículo significa devolver a la Historia su naturaleza problemática y compleja y enseñar a los estudiantes una forma de pensar el pasado más allá de la insignificancia enciclopédica de los libros de texto o de las batallas por la memoria que incendian periódicamente los medios sociales. El valor social y personal que puede aportar esta opción curricular sólo es comparable al reto y la dificultad de llevarla a la práctica en las aulas, especialmente si partimos de una cultura académica que identifica aprender historia en Secundaria como aprender una supuesta "base" de conocimientos, entendida como una enciclopedia de verdades.

\section{Conclusiones}

La multiperspectiva, la capacidad para aceptar, analizar y abrirse a múltiples versiones y perspectivas del mundo, es el corazón de la historia como disciplina, pero también el corazón de la ciudadanía democrática. Avishag Reisman y Sam Wineburg nos ofrecen la mejor definición de cómo se funden en uno estos dos corazones de la multiperspectiva:

\footnotetext{
"Proponemos que las capacidades de interpretación disciplinar histórica -es decir, la capacidad de leer e interpretar textos históricos; la capacidad de evaluar y reconciliar discursos alternativos que se reclaman como verdaderos; y la capacidad de atemperar nuestra propia prisa para emitir juicios ante visiones del mundo que compiten entre sí- constituyen el corazón de la democracia
} 
participativa. En las aulas de historia donde los estudiantes de forma regular practican estas capacidades disciplinares y afrontan las visiones del mundo anticuadas de sus predecesores, los debates de clase se constituyen como espacios para la deliberación racional. Es en estos debates en el aula donde esperamos ver a nuestros estudiantes desarrollar su propia subjetividad histórica y trascenderla en un esfuerzo de comprender a los otros" (2012, p. 172)

No hay mejor argumento para la inclusión de la multiperspectiva en el aula. No hay verdadera comprensión de la historia sin aprender a afrontar las múltiples perspectivas que conforman sus fuentes, las historias que construyen los historiadores o los usos que la sociedad hace de la historia. $Y$ tampoco podemos concebir una verdadera ciudadanía democrática sin aprender a escuchar, dialogar y valorar con las perspectivas diversas de los otros.

Existen, como hemos visto, múltiples formas de trabajar la multiperspectiva en el aula de historia, pero ninguna de ellas es fácil. Desde los estudios del célebre CHATA project no hemos dejado de comprobar la resistencia de los estudiantes para abandonar el realismo ingenuo y adentrarse en el mundo del pasado (y del presente) desde una concepción de perspectiva. No es fácil comprender y aceptar que no podemos contar el mundo "tal y como es". Asumir que existen múltiples versiones del mundo, que no es posible como historiadores, ciudadanos o personas dejar atrás nuestra particular perspectiva para contar la verdad de cómo fueron (o son) las cosas, requiere una gran energía y esfuerzo. Introducir la multiperspectiva en las aulas de historia significa apostar por un proceso difícil de aprendizaje que transformará a nuestros estudiantes, no sólo porque comprenderán la verdadera naturaleza de la historia, sino porque comprenderán un aspecto esencial del mundo social en el que viven.

\section{Agradecimientos}

Trabajo realizado en el marco de la actividad desarrollada por el Grupo de investigación ARGOS -financiado por el Gobierno de Aragón (S50_20R) y cofinanciado con FEDER 2014-2020 "Construyendo Europa desde Aragón"- y del proyecto EDU2016-78163-R "Educomunicación web 2.0 del patrimonio" (MINECO /AEI-FEDER/UE). 


\section{Referencias}

Abdou, E. D. (2017). Toward Embracing Multiple Perspectives in World History Curricula: Interrogating Representations of Intercultural Exchanges Between Ancient Civilizations in Quebec Textbooks. Theory \& Research in Social Education, 45, 378-412.

ACARA (Australian Curriculum Assessment and Reporting Authority). (2015). Australian curriculum: History: Structure. https://www.australiancurriculum.edu.au/f-10curriculum/humanities-and-social-sciences/history/structure/

Ahonen, S. (2012). Coming to Terms with a Dark Past: How Post-Conflict Societies Deal with History. Peter Lang.

Ahonen, S. (2013). Post-Conflict History Education in Finland, South Africa and BosniaHerzegovina. Nordidactica - Journal of Humanities and Social Science Education, 1, 90103.

Ahonen, S. (2017). The Lure of Grand Narratives: A Dilemma for History Teachers. En H. A. Elmersjö, A. Clark y M. Vinterek (Eds.), International Perspectives on Teaching Rival Histories. Pedagogical Responses to Contested Narratives and the History Wars (pp. 4162). Palgrave Macmillan.

Ahonen, S. (2019). The construction and deconstruction of national myths. A study of the transformation of Finnish history textbook narratives after World War II. European Politics and Society, 21(3), 341-355. https://doi.org/10.1080/23745118.2019.1645427

Alberta, Ministry of Education. (2015). Social Studies. Kindergarten to Grade 12. Alberta Ministry of Education.

Alcoe, A. (2015). Post hoc ergo propter hoc? Using causation diagrams to empower sixth-form students in their historical thinking about cause and effect. Teaching History, 161, 16-24.

Barnett, R. (2000). Realizing the university in an age of supercomplexity. The society for Research into Higher Education \& Open University Press.

Barnett, R. (2007). A Will to Learn. Being a Student in an Age of Uncertainty. SRHE \& Open University Press.

Barton, K. y McCully, A. W. (2007). Teaching controversial issues... where controversial issues really matter. Teaching History, 127, 13-19.

Barton, K. C. y McCully, A. W. (2012). Trying to "See Things Differently": Northern Ireland Students' Struggle to Understand Alternative Historical Perspectives. Theory \& Research in Social Education, 40(4), 371-408. https://doi.org/10.1080/00933104.2012.710928c

Berger, S. y Lorenz, C. (2008). Introduction: National history writing in Europe in a global age. En S. Berger y C. Lorenz (Eds.), The Contested Nation: Ethnicity, class, religion and gender in national histories (pp. 1-23). Palgrave Macmillan.

Bermúdez, A. y Argumero, D. (2018). The narrative framing of violence in teaching resources about the Spanish Conquest of America. Panta Rei. Revista Digital de Ciencia y Didáctica de la Historia, 2018, 93-118. https://doi.org/10.6018/pantarei/2018/5

Brooks, S. (2009). Historical Empathy in the Social Studies Classroom: A Review of the Literature. The Journal of Social Studies Research, 33(2), 213-234.

Carretero, M. (2001). La construcción de una identidad nacional. Cuadernos de Pedagogía, $308,52-56$.

Carretero, M. (2007). Documentos de identidad. La construcción de la memoria histórica en un mundo global. Paidós. 
Carretero, M. (2011). Comprensión y aprendizaje de la historia. En L. F. Rodríguez y N. García (Eds.), Enseñanza y aprendizaje de la Historia en la Educación Básica (pp. 69-104). Secretaría de Educación Pública, Gobierno Federal.

Carretero, M. (2011b). Constructing patriotism. Teaching history and memories in global worlds. Information Age Publishing.

Carretero, M. y Bermúdez, A. (2012). Constructing Histories. En J. Valsiner (Ed.), Oxford Habdbook of Culture and Psychology (pp. 625-646). Oxford University Press.

Carretero, M. y Kriger, M. (2008). Narrativas históricas y construcción de la identidad nacional: representaciones de alumnos argentinos sobre el "Descubrimiento" de América. Cultura y Educación, 20(2), 229-242.

Carretero, M. y Kriger, M. (2011). Historical representations and conflicts about indigenous people as national identities. Culture \& Psychology, 17(2).

Carretero, M. y Lee, P. (2014). Learning Historical Concepts. En R. K. Sawyer (Ed.), The Cambridge Handbook of the Learning Sciences (2nd ed.). Cambridge University.

Chapman, A. (2011a). Historical interpretations. En I. Davies (Ed.), Debates in History Teaching (pp. 96-108). Routledge.

Chapman, A. (2011b). Twist and Shout? Developing Sixth-Form Students' Thinking about Historical Interpretation. Teaching History, 142, 24-33.

Chapman, A. (2011c). Understanding Historical Knowing: Evidence and Accounts. En L. Perikleous y D. Shemilt (Eds.), The Future of the Past: Why History Education Matters. The Association for Historical Dialogue and Research (AHDR).

Clark, A. (2006). Teaching the nation. Politics and pedagogy in Australian history. Melbourne University Press.

Clark, A. (2009). Teaching the nation's story: comparing public debates and classroom perspectives on history education in Australia and Canada. Journal of Curriculum Studies, 41(6), 745-762. https://doi.org/10.1080/00220270903139635

Council of Europe. (2011). The Committee of Ministers to member states on intercultural dialogue and the image of the other in history teaching. https://rm.coe.int/CoERMPublicCommonSearchServices/DisplayDCTMContent?documen $\underline{\mathrm{tld}=09000016805 \mathrm{cc} 8 \mathrm{e} 1}$

Duquette, C. (2015). Relating historical consciousness to historical thinking through assessment. En K. Ercikan y P. Seixas (Eds.), New directions in assessing historical thinking (pp. 51-63). Routledge.

Elmersjö, H. A., Clark, A. y Vinterek, M. (Eds.) (2017). International Perspectives on Teaching Rival Histories. Pedagogical Responses to Contested Narratives and the History Wars. Palgrave Macmillan.

Fontana, J. (1999). Historia: análisis del pasado y proyecto social. Crítica.

Fordham, M. (2012). Out went Caesar and in came the Conqueror, though I'm sure something happened in between... Teaching History, 147, 38-46.

Foster, S. J. (1998). Politics, parallels and perennial curriculum questions: the battle over school history in England and the United States. The Curriculum Journal, 9(2), 153-164. https://doi.org/10.1080/0958517970090203

Foster, S. (2017). Teaching About the First World War in England: Exploring Controversy and Competing Historical Interpretations. En H. A. Elmersjö, A. Clark y M. Vinterek (Eds.), International Perspectives on Teaching Rival Histories. Pedagogical Responses to Contested Narratives and the History Wars. Palgrave Macmillan. 
Foster, S. J. y Rosch, R. (1997). Teaching World War I from Multiple Perspectives. Social Education, 61(7), 429-434.

Gibson, L. y Case, R. (2019). Reshaping Canadian History Education in Support of Reconciliation. Canadian Journal of Education / Revue canadienne de l'éducation, 42(1), 251-284.

González, M. F.y Carretero, M. (2013). Historical narratives and arguments in the context of identity conflicts. Estudios de Psicología, 34(1), 73-82.

Grever, M. (2012). Dilemmas of Common and Plural History. En M. Carretero, M. Asensio y M. Rodríguez-Moneo (Eds.), History Education and the Construction of National Identities (pp. 75-91). Information Age Publishing, Inc.

Hakoköngäs, E., Kleemola, O., Sakki, I. y Kivioja, V. (2020). Remembering war through images: Visual narratives of the Finnish Civil War in history textbooks from the 1920s to the 2010s. Memory Studies, 00, 1-16. https://doi.org/10.1177/1750698020959812

Huijgen, T., van Boxtel, C., van de Grift, W. y Holthuis, P. (2016). Toward Historical Perspective Taking: Students' Reasoning When Contextualizing the Actions of People in the Past,. Theory \& Research in Social Education, 00, 1-35. https://doi.org/10.1080/00933104.2016.1208597

Keet, P. y Datema-Chang, H. (2020). Multiperspectiviteit bij sensitief erfgoed in de klas. Tijdschrift voor lerarenopleiders, 41(3), 166-175.

Klein, S. R. E. (2010). Teaching History in the Netherlands: Teachers' Experiences of a Plurality of Perspectives. Curriculum Inquiry, 40(5), 614-634.

Korostelina, K. V. y Lässig, S. (Eds.) (2013). History Education and Post-Conflict Reconciliation: Reconsidering Joint Textbook Projects. Routledge.

Kropman, M., van Drie, J. y van Boxtel, C. (2019). Multiperspectivity in the History Classroom: The Role of Narrative and Metaphors. En M. Hanne y A. A. Kaal (Eds.), Narrative and Metaphor in Education: Look Both Ways (pp. 63-75). Routledge.

Kropman, M., van Boxtel, C. y van Drie, J. (2020). Narratives and Multiperspectivity in Dutch Secondary School History Textbooks. Journal of Educational Media, Memory, and Society, 12(1), 1-23. https://doi.org/10.3167/jemms.2020.120101

Lee, P. J. (1998). 'A lot of guess work goes on'. Children's understanding of historical accounts. Teaching History, 92, 29-36.

Lee, P. J. (2005). Putting Principles into Practice: Understanding History. En M. S. Donovan y J. D. Bransford (Eds.), How students learn: history, mathematics, and science in the classroom (pp. 31-77). The National Academies Press.

Lee, P. J. y Ashby, R. (2000). Progression in Historical Understanding among Students Ages 714. En P. Stearns, P. Seixas y S. Wineburg (Eds.), Knowing, Teaching Learning History (pp. 199-223). New York University Press.

Lee, P. y Ashby, R. (2001). Empathy, perspective taking, and rational understanding. En O.L. Davis Jr., E. A. Yeager y S. J. Foster (Eds.), Historical Empathy and Perspective Taking in the Social Studies (pp. 21-50). Rowman \& Littlefield Publishers, Inc.

Lee, P. J. y Shemilt, D. (2003). A scaffold, not a cage: Progression and progression models in history. Teaching History, 113, 13-23.

Lee, P. J. y Shemilt, D. (2004). 'I just wish we could go back in the past and find out what really happened': progression in understanding about historical accounts. Teaching History, 117, 25-31.

Levstik, L. S. (1997). "Any History is Someone's History". Listening to Multiple Voices from the Past. Social Education, 61(1), 48-51. 
Lipman, M. y Sharp, A. M. (2002). La filosofía en el aula. Ediciones de la Torre (orig.: Temple University Press, 1980).

Marcus, A. S. y Stoddard, J. D. (2009). The Inconvenient Truth about Teaching History with Documentary Film: Strategies for Presenting Multiple Perspectives and Teaching Controversial Issues. The Social Studies, 100(6), 279-284. https://doi.org/10.1080/00377990903283957

Mark, E. (2017). Teaching the Ends of Empires. EuroClio. https://www.euroclio.eu/ tag/teachingmultiperspectivity/

Nygren, T., Vinterek, M., Thorp, R. y Taylor, M. (2017). Promoting a Historiographic Gaze through Multiperspectivity in History Teaching. En H. A. Elmersjö, A. Clark y M. Vinterek (Eds.), International Perspectives on Teaching Rival Histories. Pedagogical Responses to Contested Narratives and the History Wars (pp. 207-228). Palgrave Macmillan.

Nordgren, K. y Johansson, M. (2015). Intercultural historical learning: a conceptual framework. Journal of Curriculum Studies, 47(1), 1-25. https://doi.org/10.1080/ $\underline{00220272.2014 .956795}$

Paricio, J. (2018). El valor de la historia. Estudio de alternativas curriculares en Secundaria (1): una visión integrada de las transformaciones (cambio/continuidad) que conducen y modelan el presente (conciencia histórica). CLIO. History and History teaching, 44, 232247. http://clio.rediris.es/n44/articulos/14Paricio2018.pdf

Paricio, J. (2019). El valor de la historia. Estudio de alternativas curriculares en Secundaria (2): aprender sobre la alteridad y la naturaleza humana a través de la empatía o toma de perspectiva histórica. CLIO. History and History teaching, 43.

Paricio, J. (2021). Perspective as a threshold concept for learning history. History Education Research Journal, 18(1) (en prensa).

Paul, R. y Elder, L. (2006). The Thinker's Guide To Fallacies: The Art of Mental Trickery and Manipulation. The Foundation for Critical Thinking.

Pozo, J. I. y Carretero, M. (1989). Las explicaciones causales de expertos y novatos en Historia. En M. Carretero, J. I. Pozo y M. Asensio (Eds.), La enseñanza de las ciencias sociales (pp. 139-164). Visor.

Reisman, A. y Wineburg, S. (2008). Teaching the Skill of Contextualizing in History. The Social Studies, 99(5), 202-207.

Rüsen, J. (2004). Historical consciousness: Narrative structure, moral function, and ontogenetic development. En P. Seixas (Ed.), Theorizing historical consciousness (pp. 63-85). University of Toronto Press. (Original: 1989)

Rüsen, J. (2008). History: Narration, Interpretation, Orientation (2nd ed.). Berghan Books.

Rüsen, J. (2012). Tradition: a principle of historical sense-generation and its logic and effect in historical culture. History and Theory, 51(4), 45-59. https://doi.org/doi:10.1111/j.14682303.2012.00646.x

Schnakenberg, U. (2010). Developing multiperspectivity through cartoon analysis: strategies for analysing different views of three watersheds in modern German history. Teaching History, 139, 32-39.

Schuitema, J. (2008). Talking about values: a dialogic approach to citizenship education as an integral part of history classes. Universiteit van Amsterdam.

Scott, D. (2013). Teaching Aboriginal Perspectives: An Investigation into Teacher Practices amidst Curriculum Change. Canadian Social Studies, 46(1), 31-43.

Seixas, P. (2000). Schweigen! die Kinder! or, Does Postmodern History Have a Place in the Schools? In P. N. Stearns, P. Seixas y S. S. Wineburg (Eds.), Knowing, teaching and 
learning history. National and international perspectives (pp. 19-37). New York University Press.

Seixas, P. (2005). Historical Consciousness: the Progress of Knowledge in a Postprogressive Age. En J. Straub (Ed.), Narration, Identity, and Historical Consciousness. Berghahn Books.

Seixas, P. (2007). Who needs a canon?, en M. Grever y S. Stuurman (Eds.), Beyond the canon. History for the twenty-first century (pp. 19-30). Palgrave Macmillan.

Seixas, P., Fromowitz, D. y Hill, P. (2005). History, Memory and Learning to Teach. En R. Ashby, P. Gordon y P. Lee (Eds.), Understanding History. Recent Research in History Education. Volume 4. Routledge.

Sharp, H. (2017). After the Ideological Battles: Student Views on Sources Representing the Gallipoli Conflict. En H. A. Elmersjö, A. Clark, y M. Vinterek (Eds.), International Perspectives on Teaching Rival Histories. Pedagogical Responses to Contested Narratives and the History Wars (pp. 229-250). Palgrave Macmillan.

Stoel, G. L., van Drie, J. P. y van Boxtel, C. A. M. (2015). Teaching towards historical expertise: developing a pedagogy for fostering causal reasoning in history. Journal of Curriculum Studies, 47(1), 49-76. doi:10.1080/00220272.2014.968212

Stradling, R. (2003). Multiperspectivity in history teaching: a guide for teachers. Council of Europe.

Swedish National Agency for Education. (2018). Curriculum for the compulsory school, preschool class and school-age educare. Revised 2018. Swedish National Agency for Education.

Van Nieuwenhuyse, K. y Wils, K. (2012). Remembrance education between history teaching and citizenship education. Citizenship Teaching \& Learning, 7(2), 157-171. doi:10.1386/ctl.7.2.157_1

VanSledright, B. (2002). In Search of America's Past. Learning to Read History in Elementary School. Teachers College Press, Columbia University

VanSledright, B. (2011). The challenge of rethinking history education: On practices, theories, and policy. Routledge.

Waters, D. (2014). A host of histories: helping Year 9s explore multiple narratives through the history of a house. Teaching History, 156, 42-49.

Wineburg, S. (1991). On the Reading of Historical Texts: Notes on the Breach Between School and Academy. American Educational Research Journal, 28(3), 495-519.

Wineburg, S. (2001). Historical Thinking and Other Unnatural Acts: Charting the Future of Teaching the Past. Temple University Press.

Wineburg, S. (2010). Thinking Like a Historian. Teaching with Primary Sources Quarterly, 3(1), 2-4. 